\title{
The Effect of Ultrasonic Additive Manufacturing on Integrated Printed Electronic Conductors
}

\author{
Alkaios Bournias-Varotsis ${ }^{1}$. Shanda Wang ${ }^{1} \cdot$ David Hutt $^{1}$ - Daniel S. Engstrøm ${ }^{1}$
}

Received: 19 October 2017 / Accepted: 7 February 2018 / Published online: 13 March 2018

(C) The Author(s) 2018

\begin{abstract}
Ultrasonic additive manufacturing (UAM) is a low temperature manufacturing method capable of embedding printed electronics in metal components. The effect of UAM processing on the resistivity of conductive tracks printed with five different conductive pastes based on silver, copper or carbon flakes/particles in either a thermoplastic or thermoset filler binder are investigated. For all but the carbon-based paste, the resistivity changed linearly with the UAM energy input. After UAM processing, a resistivity increase of more than 150 times was recorded for the copper based thermoset paste. The silver based pastes showed a resistivity increase of between 1.1 and 50 times from their initial values. The carbon-based paste showed no change in resistivity after UAM processing. Focussed ion beam microstructure analysis of the printed conductive tracks before and after UAM processing showed that the silver particles and flakes in at least one of the pastes partly dislodged from their thermoset filler creating voids, thereby increasing the resistivity, whereas the silver flakes in a thermoplastic filler did not dislodge due to material flow of the polymer binder. The lowest resistivity $\left(8 \times 10^{-5} \Omega \mathrm{cm}\right)$ after UAM processing was achieved for a thermoplastic paste with silver flakes at low UAM processing energy.
\end{abstract}

\section{Graphical Abstract}

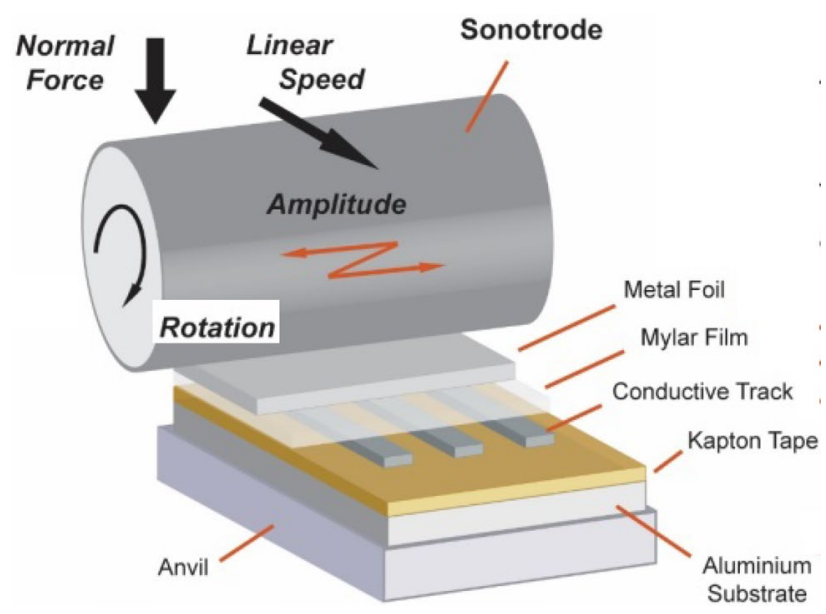

Keywords Ultrasonic additive manufacturing · Printed electronics · Isotropic conductive adhesives · Ultrasonic energy · Embedded electronics

Daniel S. Engstrøm

d.engstrom@lboro.ac.uk

1 Wolfson School of Mechanical, Electrical and Manufacturing Engineering, Loughborough University, Loughborough LE11 3TU, UK

\section{Introduction}

Embedding printed electronics in components can lead to added functionality such as sensing or monitoring [1]. Embedding electronics in metal components, however, 
is inherently difficult due to the high temperatures often required during metal processing. Ultrasonic additive manufacturing (UAM) is a hybrid sheet lamination manufacturing technology that enables the fabrication of metal parts through subsequent and repeated additive and subtractive steps [2]: thin metal foils are bonded layer-by-layer during the ultrasonic metal welding (UMW) step and the desired shape is given to the part by periodic CNC machining. Aluminium is the most commonly used UAM material due to its compatibility with the UMW step: high quality bonds can be achieved at relatively low ultrasonic energy levels. During the bonding step, a cylindrical tool head with a textured surface, called the sonotrode, is pressed against the top surface of the processed metal foil with an exerted force $(F)$. The sonotrode is then rolled over the foil with a linear speed $(\mathrm{S})$, while it oscillates at an ultrasonic frequency $(20 \mathrm{kHz})$ at a pre-set amplitude (A) perpendicular to the direction of rolling. This process causes the mating surfaces at the foilfoil interface to come in close metal contact and form solid state metal bonds $[3,4]$.

Bonding in UAM occurs in the presence of a high degree of plastic metal flow, due to the ultrasonic softening phenomenon [4]. This allowed researchers in the past to create composite metal matrix structures with embedded functional materials and other smart features, such as optical fibres [5, 6], shape memory alloy fibres [7, 8], other shape memory and magnetostrictive materials for embedded sensing applications [9] and smart switches for a structural antenna [10].

The temperatures developed during the solid-state UMW step are considerably lower than the melting point of the processed material. For aluminium alloys, temperatures peak at just under $200{ }^{\circ} \mathrm{C}$ for very high power UAM processing parameters [11] and the heat dissipates very rapidly during processing $[11,12]$. This has allowed researchers to embed printed conductive materials and other pre-packaged electronic sensors into an aluminium matrix. For example, Robinson et al. [13] showcased a method of dispensing and embedding printed conductive pathways into CNC machined pockets of a UAM fabricated substrate and Siggard et al. [14] embedded a pre-packaged thermal sensor following a similar methodology. Li et al. [15, 16] embedded screen printed insulating materials and conductive pathways directly between the foil-foil interface, demonstrating that a machining step is not necessary for embedding such materials in the metal matrix.

The aforementioned studies used isotropic conductive adhesives (ICAs) (commonly referred to as conductive pastes) to create the electrically conductive pathways. ICAs consist of particles or flakes of electrically conductive filler material (typically silver, copper, gold or carbon) that are dispersed in a polymer adhesive binder matrix. The polymer binder is typically a thermoset, but thermoplastic adhesives are also used. After the curing or drying of the polymer binder, conductivity is achieved when the composite is loaded with a sufficiently high amount of conductive filler material (above the percolation threshold). When this is achieved, the conductive particles come in close contact and a network of electrical pathways of low resistance is created in the material. The adhesive matrix plays an important role in the process as it ensures that sufficient pressure is exerted between the conductive particles and provides mechanical integrity to the structure. In all studies of UAM that used ICAs, a change in the resistivity of the conductive tracks was reported after the UMW step. A preliminary study also confirmed that the ultrasonic energy introduced to the ICA tracks during UAM treatment has a negative effect on the conductive properties of the materials [17]. Nevertheless, the evidence given in prior works concerning this phenomenon are inconclusive due to the small number of ICA materials examined.

In this paper, the results of a study examining the effects of ultrasonic energy used in the UAM process on the conductive properties of ICAs are presented. A wide range of materials and processing parameters were used and the conductive tracks were assessed in terms of resistivity, dimensional deformation and microstructural change, in order to identify the underlying mechanism. The conductive adhesives used had fundamentally different characteristics in terms of binder material (thermoset or thermoplastic) and conductive filler (silver, copper and carbon). Percolation theory [18-20] was used to rationalize the observed resistivity changes. This work will aid future researchers and engineers with the selection of the ICAs that are most compatible with the UAM process.

\section{Materials and Experimental Procedure}

\subsection{Conductive Pastes}

The five electrically conductive pastes investigated here were the commercially available Gwent C2110817D5, Epo-tek E4110-PFC, Gwent C2131014D3, Gwent C2050503P1 and an experimental copper particle paste similar in nature to that described in [21]. In this paper, they are designated with the code names Ag-TP, Ag-TS1, Ag-TS2, C-TS and Cu-TS, respectively. Their material characteristics are summarized in Table 1. In this table, the material (i.e. silver, copper or carbon) and shape (i.e. particles or flakes) of the conductive filler and the type of the polymer binder (i.e. thermoset (TS) or thermoplastic (TP)) is indicated. The resistivity range (specified by the manufacturers) for the pastes used in this study, is typical for the industry with a resistivity $50-300$ times that of bulk silver $\left(1.59 \times 10^{-6} \Omega \mathrm{cm}\right)$ and copper $\left(1.68 \times 10^{-6} \Omega \mathrm{cm}\right)$. 
Table 1 Material properties of the five investigated pastes as stated by the manufacturers

\begin{tabular}{|c|c|c|c|c|c|}
\hline & Ag-TP & Ag-TS1 & Ag-TS2 & C-TS & $\mathrm{Cu}-\mathrm{TS}$ \\
\hline Product Code & $\begin{array}{l}\text { Gwent C2110817D5 } \\
\text { [22] }\end{array}$ & $\begin{array}{l}\text { Epo-Tek E4110-PFC } \\
\text { [23] }\end{array}$ & $\begin{array}{l}\text { Gwent C2131014D3 } \\
\text { [24] }\end{array}$ & $\begin{array}{l}\text { Gwent C2050503P1 } \\
\text { [25] }\end{array}$ & N/A \\
\hline Filler & Silver flakes & $\begin{array}{l}\text { Silver particles and } \\
\text { flakes }\end{array}$ & Silver flakes & $\begin{array}{l}\text { Carbon powder and } \\
\text { graphite flakes }\end{array}$ & Copper particles \\
\hline Binder & $\begin{array}{l}\text { Single component } \\
\text { thermoplastic (Poly- } \\
\text { ester) }\end{array}$ & $\begin{array}{l}\text { Two component ther- } \\
\text { moset (Epoxy) }\end{array}$ & $\begin{array}{l}\text { Single component } \\
\text { thermoset (Epoxy) }\end{array}$ & $\begin{array}{l}\text { Single component } \\
\text { thermoset (Epoxy) }\end{array}$ & $\begin{array}{l}\text { Single component ther- } \\
\text { moset (Epoxy) }\end{array}$ \\
\hline Particle size & $\leq 17 \mu \mathrm{m}$ & $\leq 20 \mu \mathrm{m}$ & $\leq 17 \mu \mathrm{m}$ & $\begin{array}{l}\leq 1 \mu \mathrm{m} \text { (carbon) } \\
\leq 15 \mu \mathrm{m} \text { (graphite) }\end{array}$ & $14-25 \mu \mathrm{m}$ \\
\hline Solids content & $64.5-65.5 \mathrm{wt} \%$ & Approx. $70 \mathrm{wt} \%$ & $57-59.75 \mathrm{wt} \%$ & $49.2-55 \mathrm{wt} \%$ & $83.3 \mathrm{wt} \%$ \\
\hline Viscosity & 22-27 Pa s & $50-60 \mathrm{~Pa} \mathrm{~s}$ & $6.5-8.5 \mathrm{~Pa} \mathrm{~s}$ & $5.2-9.8 \mathrm{~Pa} \mathrm{~s}$ & Not available \\
\hline Volume resistivity & $7.5 \times 10^{-5} \Omega \mathrm{cm}$ & $50 \times 10^{-5} \Omega \mathrm{cm}$ & $25 \times 10^{-5} \Omega \mathrm{cm}$ & $125 \times 10^{-3} \Omega \mathrm{cm}$ & $9.0 \times 10^{-5} \Omega \mathrm{cm}$ \\
\hline Curing conditions & $\begin{array}{l}150^{\circ} \mathrm{C} \text { for } 30 \mathrm{~min} \text { in } \\
\text { box oven }\end{array}$ & $\begin{array}{l}120^{\circ} \mathrm{C} \text { for } 30 \mathrm{~min} \text { in } \\
\text { box oven }\end{array}$ & $\begin{array}{l}120^{\circ} \mathrm{C} \text { for } 10 \mathrm{~min} \text { in } \\
\text { box oven }\end{array}$ & $\begin{array}{l}150^{\circ} \mathrm{C} \text { for } 45 \mathrm{~min} \text { in } \\
\text { box oven }\end{array}$ & $\begin{array}{c}\text { Thermal cure to } 150^{\circ} \mathrm{C} \\
\text { on a hotplate in an } \\
\text { argon atmosphere }\end{array}$ \\
\hline
\end{tabular}

Table 2 Dispensing parameters used for the five pastes

\begin{tabular}{lrrrrr}
\hline & Ag-TP & Ag-TS1 & Ag-TS2 & C-TS & Cu-TS \\
\hline Nozzle Dia. $(\mu \mathrm{m})$ & 150 & 330 & 250 & 150 & 330 \\
Pressure $(\mathrm{kPa})$ & 250 & 500 & 235 & 250 & 500 \\
Speed $(\mathrm{mm} / \mathrm{s})$ & 3 & 1 & 3 & 3 & 1 \\
Print gap $(\mu \mathrm{m})$ & 100 & 100 & 80 & 120 & 150 \\
Pitch $(\mu \mathrm{m})$ & 250 & 250 & 350 & 350 & 350 \\
\hline
\end{tabular}

\subsection{Sample Preparation and Syringe Dispensing}

Substrates were prepared by first cutting a $1.5 \mathrm{~mm}$ thick aluminium Al1050 sheet to size (approx. $80 \mathrm{~mm}$ by $25 \mathrm{~mm}$ ) and then coating it with a layer of self-adhesive polyimide tape (75 $\mu \mathrm{m}$ thick Kapton ${ }^{\mathrm{TM}}$ tape). This aluminium substrate is commonly used with UAM $[6-8,26]$ and the polyimide tape has excellent insulating, mechanical and thermal properties. The polyimide tape also provides an excellent surface for the dispensing of the conductive materials and past research has shown that it can withstand the mechanical loads introduced during UAM [10].

For the dispensing of the conductive material, a Musashi Shotmaster 500 system (Musashi Engineering Inc., Japan) was used. Different nozzle sizes and dispensing parameters (Table 2) were used for each material as the conductive materials had widely variable flowability characteristics. $60 \mathrm{~mm}$ long tracks were printed by following a meander path as shown in Fig. $1 \mathrm{~b}$ instead of single lines. This was done to ensure the resulting patterns had similar overall width $(1.4-1.8 \mathrm{~mm})$ and reduced the effects of the semi-elliptical cross-section that was characteristic of the single tracks resulting in a more rectangular profile (Fig. 1c). Apart from the semi-elliptical shape, narrower tracks similar to those found (a)

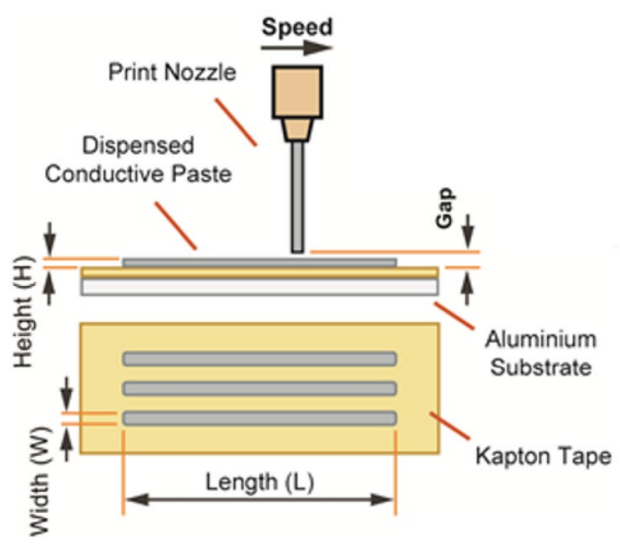

(b)

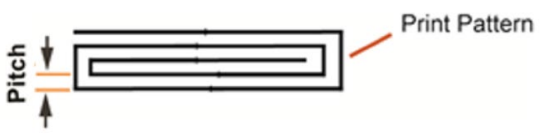

(c)

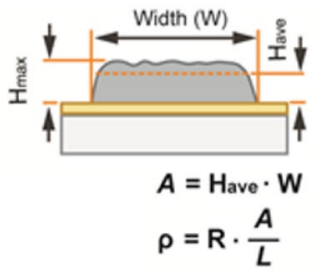

Fig. 1 Illustration of the syringe dispensing process of the printed conductive tracks: a the sample and syringe dispensing system with key dimensions, $\mathbf{b}$ the printing pattern used to create each track, $\mathbf{c}$ the cross-sectional area measured for the resistivity calculation

in printed electronics, are not expected to respond differently to the UAM treatment and have previously been tested with similar results $[15,17]$. The samples were cured according to the manufacturer's recommendations (see Table 1) and their dimensions were measured after curing using an optical 
measuring system (Talysurf CLI 2000, Taylor Hobson Ltd, UK). Three tracks were printed on each substrate and were later ultrasonically treated simultaneously. Figure 1 illustrates the printing process and the samples produced.

The ultrasonic energy was applied to the conductive material using the UAM Alpha 2 welding machine [3, 5-8, 15, 16, 26, 27] as illustrated in Fig. 2. A protective polyester BoPET polymer film ( $75 \mu \mathrm{m}$ thick Mylar ${ }^{\mathrm{TM}}$ film) was placed on top of the conductive tracks and an aluminium foil (100 $\mu \mathrm{m}$ thick Al3003-H18 foil) was placed between the protective polymer film and the sonotrode. These two layers were used (i) to protect the conductive tracks from coming into direct contact with the rough textured sonotrode, (ii) to facilitate the propagation of the ultrasonic oscillation from the sonotrode to the conductive material, (iii) to simulate the embedding process, (iv) to prevent permanent bonding of the $\mathrm{Al}$ foil layers thereby allowing them to be separated and examined after processing.

The ultrasonic energy was controlled by altering the amplitude of the ultrasonic oscillations (A) and the normal force (F) applied by the sonotrode. For simplicity, the linear speed (S) was kept constant. The total ultrasonic energy input $\left(\mathrm{E}_{\mathrm{t}}\right)$ into the top surface of the protective polymer film was estimated using the analytical formula of Yang et al. [28]. Three sets of processing parameters were selected: a low, a medium and a high (as shown in Table 3 ). The levels of the amplitude and the force were chosen accordingly, so that the processing parameters were within the ultrasonic welding window for Al1050 (a material commonly used with UAM) and so that the ultrasonic energy input increased linearly (from approx. 0.6-1.8 J) between the three treatment levels. Moreover, in order to examine the effect of the rolling motion of the sonotrode on the dimensions and resistivity of the conductive tracks, control samples were subjected to a roll-only treatment, using processing parameters similar to the medium treatment setting, but without inducing any ultrasonic vibration.
Table 3 Parameters applied during UAM processing

\begin{tabular}{lllll}
\hline & Low & Medium & High & Roll \\
\hline Amplitude (A) & $16 \mu \mathrm{m}$ & $18 \mu \mathrm{m}$ & $22 \mu \mathrm{m}$ & 0 \\
Force (F) & $1000 \mathrm{~N}$ & $1400 \mathrm{~N}$ & $1600 \mathrm{~N}$ & $1400 \mathrm{~N}$ \\
Speed (S) & $30 \mathrm{~mm} / \mathrm{s}$ & $30 \mathrm{~mm} / \mathrm{s}$ & $30 \mathrm{~mm} / \mathrm{s}$ & $30 \mathrm{~mm} / \mathrm{s}$ \\
Total energy $\left(\mathrm{E}_{\mathrm{t}}\right)$ & $0.662 \mathrm{~J}$ & $1.234 \mathrm{~J}$ & $1.843 \mathrm{~J}$ & - \\
\hline
\end{tabular}

For the Ag-TP, Ag-TS2 and C-TS materials, thirty tracks in total (i.e. ten substrates with three tracks on each substrate) were prepared and treated: nine tracks (three substrates) were treated at each ultrasonic energy level (i.e. low, medium or high energy) and three tracks (one substrate) were subjected to roll only. Due to their very high viscosity, the precise dispensing of the Ag-TS1 and the Cu-TS pastes was difficult. Moreover, in the case of the copper paste, the curing conditions and the small availability of the material did not allow for the preparation of a large number of samples. For these two materials, only twelve tracks were prepared and treated (i.e. four substrates in total), resulting in the treatment of three tracks at each ultrasonic energy level and three tracks at roll only. Extra control samples were also prepared for microstructural analysis and cross sectioning.

\subsection{Resistivity Measurements}

The resistance of the tracks was measured using a bespoke 4-point probe setup and a Keithley 2425 tabletop multimeter. Six resistance measurements of each track were taken and the results were averaged. The distance between the sensing probes was measured to be $L=44.704 \pm 0.001 \mathrm{~mm}$ (SmartScope Flash 200, Optical Gaging Products Inc., NY). Spring-loaded, gold-coated Harwin probes with convex heads were used for the sensing probes and with serrated heads for the current leads.
Fig. 2 Illustration of experimental setup for UAM processing of the conductive tracks

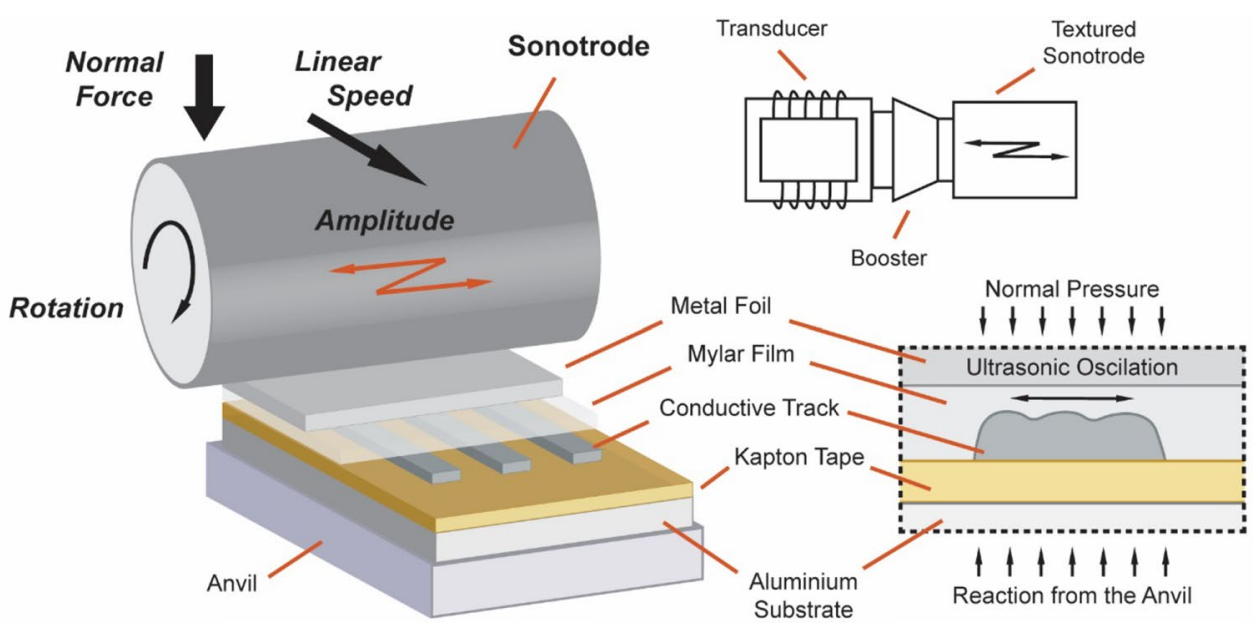


The resistivity of the tracks was calculated before and after the ultrasonic treatment from the resistance measurements using Pouillet's law: $\rho=R \cdot A / L$ where $R$ is the resistance of the material, $L$ is the length between the measuring probes and $A$ is the cross-sectional area of the conductor. The cross-sectional area was measured using an optical measuring system (Talysurf CLI 2000, Taylor Hobson Ltd, UK) and at six locations along the length of each track.

\subsection{Microstructure Characterization}

For each conductive material, a typical track treated at the medium ultrasonic setting and an untreated control sample were examined using FIB-SEM imaging. The samples were first cut to size, mounted on sample holders using silver adhesive and coated with a thin Pt-Au layer to avoid charging. A focused ion beam (FIB) was used to cross-section the prepared samples and scanning electron microscopy (SEM) was employed for the imaging. SEM was also utilized for imaging the surface morphology of the tracks. The results were analyzed quantitatively (i.e. particle size and particle count measurements) using open source image processing software (ImageJ).

A number of representative samples were also cross sectioned using a low speed saw, mounted and polished to a $1 \mu \mathrm{m}$ finish using an automatic polishing machine, and examined under an optical microscope (Eclipse MA200 inverted microscope, Nikon Metrology). Optical imaging was not sufficient to record changes in the microstructure of the materials, but it was used to verify the dimensional measurements of the printed tracks as well as other macroscopic changes of the morphology of the tracks. The samples examined with both FIB-SEM and optical imaging were cross-sectioned along their width in an area close to the middle of the tracks.

\section{Results and Discussion}

\subsection{Morphology Change}

The printed and cured tracks of each conductive paste were uniform in size and shape prior to the UAM processing with a variation in the average cross-sectional area of less than $1 \%$ for each material. An example of the printed tracks can be seen in Fig. 3. Due to a large difference in the viscocity of the individual pastes, which affects the flow of the paste once dispensed, the average height and width of the printed tracks varied. Ag-TP and C-TS had the smallest dimensions with a layer height close to $20 \mu \mathrm{m}$, while the tracks printed using Ag-TS1 and Cu-TS had the largest heights of up to approx. $160 \mu \mathrm{m}$ (the maximum height of the tracks was approximately $5-10 \%$ larger). The individual values are given in Table 4 and the definition of the parameters is shown in Fig. 1.

The dimensions of the conductive tracks were altered after processing in the UAM equipment, proving that the conductive material was subjected to mechanical deformation. The average height, width and cross-sectional area can be seen in Fig. 4 for three separate conditions: (1) the printed tracks after curing; (2) after UAM processing (all three ultrasonic processing levels); (3) after rolling the sonotrode over
Fig. 3 Photographs and SEM images of the top view of a representative $\mathrm{Ag}$-TP sample a as-printed and cured and $\mathbf{b}$ after ultrasonic treatment at medium setting

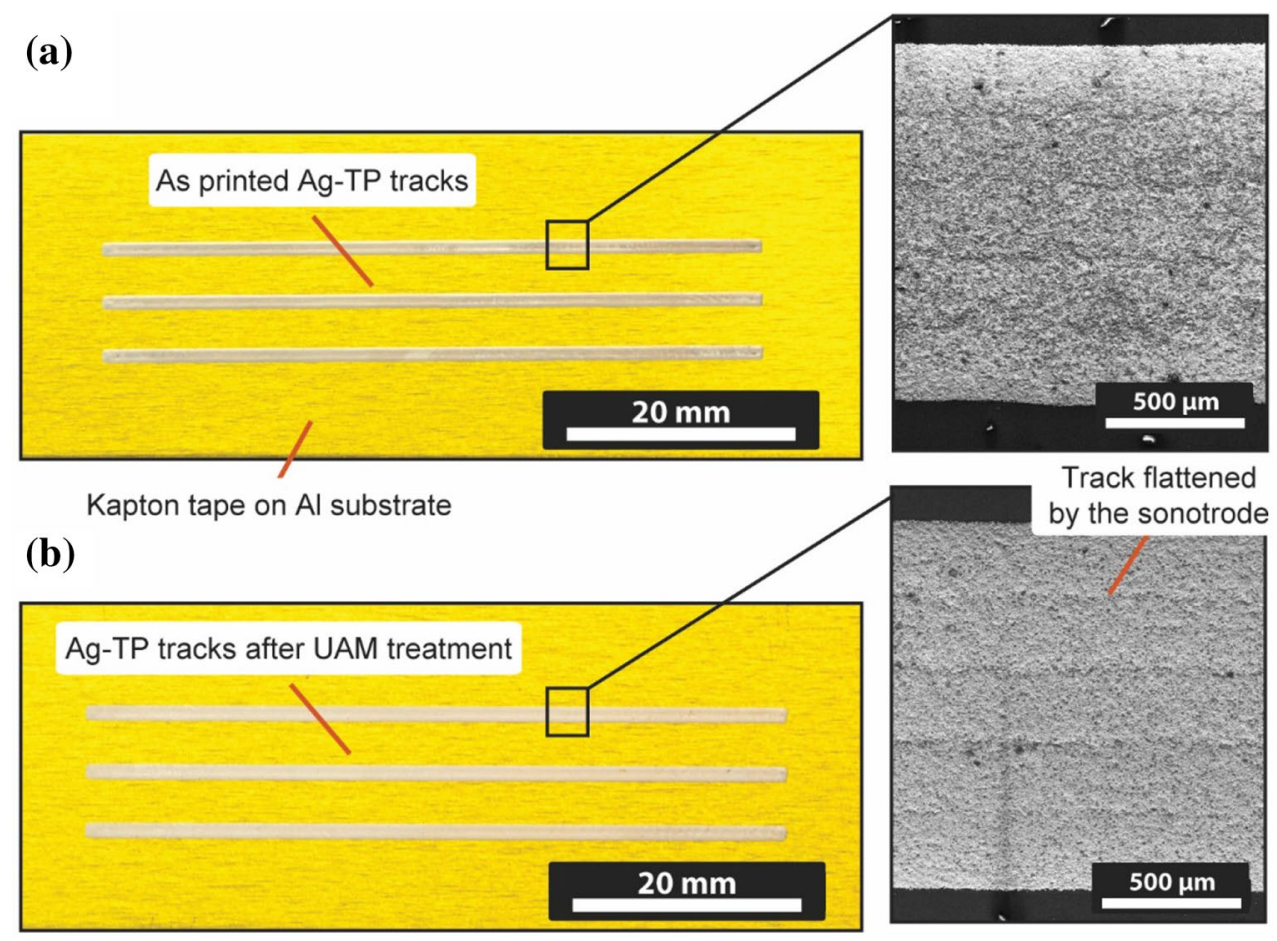


the tracks with no ultrasound applied. The experiment for rolling without ultrasound applied was conducted to separate the effect of ultrasound from the pressure alone. Both the roll only and full UAM process deformed the printed tracks resulting in a reduced height, wider tracks and a reduced cross-sectional area. When comparing the morphology change of the tracks during rolling and UAM processing, it is noteworthy that the area reduction for Ag-TS1 and Ag-TS2 is significantly smaller for UAM processing than for roll only. This is despite a significant flow of the material when ultrasound is also applied as seen by the increase in the width of the tracks. Notably, there was no correlation between the dimensional change and the level of ultrasonic treatment.

\subsection{The Effect of UAM on the Resistivity of the Conductive Tracks}

For every paste, the average resistivity of the dispensed tracks after conventional heat treatment but before ultrasonic treatment was within the range set by the manufacturer or, in the case of $\mathrm{Cu}$-TS, comparable to similar materials used in previous work [21]. The average resistivity of the tracks for all five pastes is summarized in Fig. 5a-e for five cases: low, medium and high UAM energy, roll without ultrasound and a reference before treatment. The error bars indicate the standard deviation. All data points fell within 2 standard deviations with the exception of a single outlier for $\mathrm{Cu}$-TS (low) which did not distort the overall trends. For Ag-TS1 and Cu-TS a smaller number of datasets were available, but the maximum and minimum values for the smaller datasets $( \pm 15 \%)$ were less than the variation for the larger datasets $( \pm 40 \%)$. These relatively large variations should be compared to the orders of magnitude change in resistivity when various levels of UAM energy were applied.

The resistivity of the conductive tracks that were subjected to rolling only decreased for all materials, apart from $\mathrm{Cu}-\mathrm{TS}$, which increased by a factor of 30 . No significant change in the resistance of the other samples was recorded, but, as mentioned previously, their average cross-sectional areas were decreased leading to the apparent decrease in resistivity. In the case of $\mathrm{Cu}$-TS, the observed increase in resistivity after rolling alone suggests that this material is unsuitable for withstanding mechanical loading without significant change in its conductive properties. This point will be revisited in the microstructure analysis but previous research has determined that many printed conductive materials have a limited capability to withstand mechanical deformation before their electrical properties degrade [29-31].

The resistivity change for each conductive material due to UAM processing varied greatly (see Fig. 5). The materials 

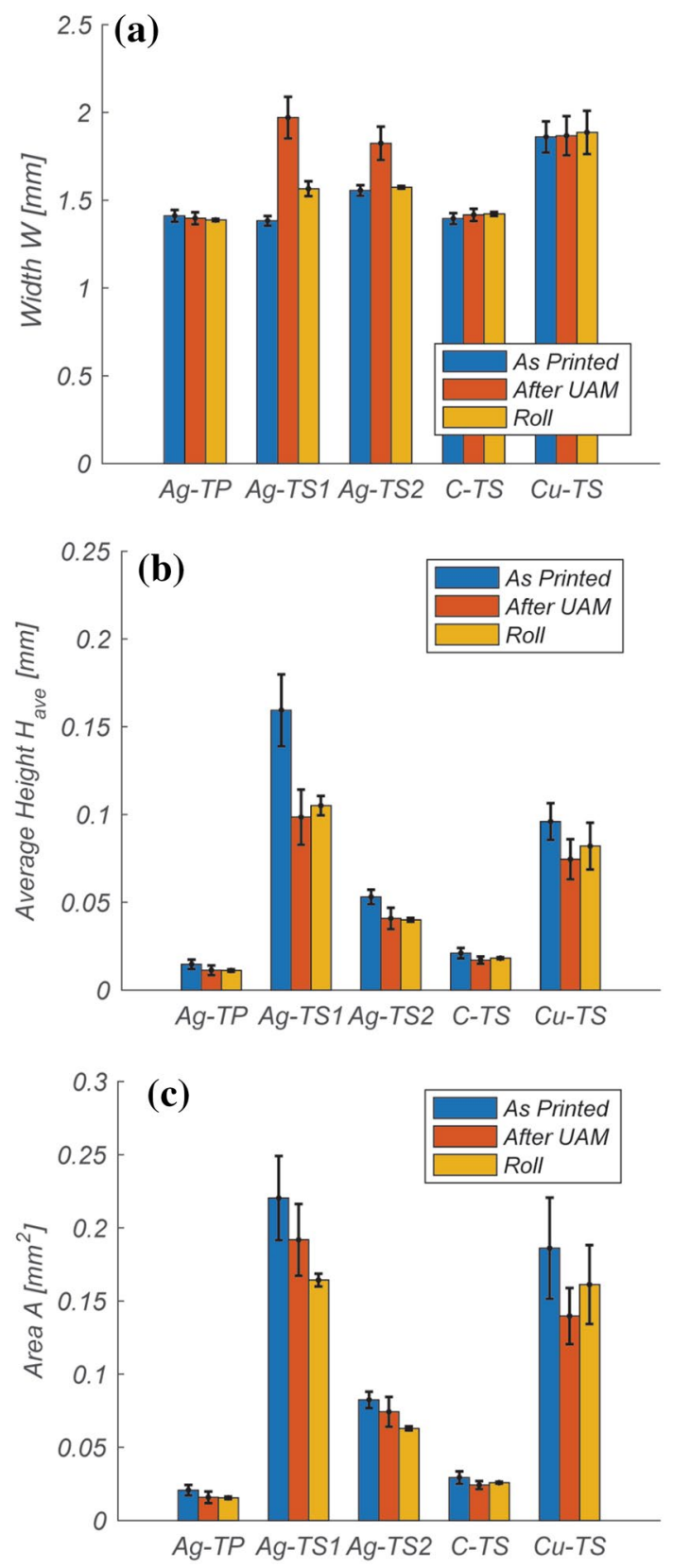

Fig. 4 a Width, b height and $\mathbf{c}$ cross-sectional area of the printed conductive tracks for three cases; as printed, after UAM and after the sonotrode rolled over the tracks with no ultrasound

Ag-TS1, Ag-TS2 and Cu-TS all had a large increase in resistivity, Ag-TP only had a moderate increase in resistivity, whereas the resistivity of C-TS was almost unaffected by the ultrasonic treatment. The initial solid loading of conductive particles in the ICAs ranged from 49.2 to $83.3 \mathrm{wt} \%$ but there was no correlation between the resistivity (before or after processing) and the solid loading.

In Fig. 6a, b the resistivity increase (i.e. the resistivity after treatment divided by the initial resistivity) for each conductive material is plotted against the total ultrasonic energy input. The resistivity of the conductive tracks increased almost linearly with the application of higher ultrasonic energy for the silver and copper loaded materials, while the ultrasonic treatment had almost no effect on the resistivity of the carbon/graphite loaded tracks. The rate of resistivity increases also varied widely for each material. For example, the resistivity of the Ag-TP tracks increased by approx. 50\% at the highest ultrasonic treatment setting, while the resistivity of the Ag-TS2 and Cu-TS tracks increased by about 2 orders of magnitude. The difference in the resistivity changes for roll only and UAM processing indicates that the observed resistivity changes were caused mainly by the ultrasonic treatment and not the rolling effect of the sonotrode.

Conductive tracks of Ag-TS2 have recently been embedded by ultrasonic additive manufacturing by Li et al. [16] and a resistivity increase of up to $15 \times$ after UAM processing was reported. Although the resistivity change is significantly smaller than the values reported in this study, the processing conditions were not identical and the trend supports our findings.

The deformation of the printed tracks during UAM processing plays an important role in the resistivity change since the initial thickness of the tracks affects the resistivity change. To examine this effect, several layers of Ag-TP were printed on top of each other to create tracks of varied thickness and their resistivity change after UAM treatment at medium ultrasonic setting was recorded. Figure 7 shows the resistivity change (i.e. the resistivity after treatment divided by the initial resistivity) for tracks with 1, 2 and 3 layers of Ag-TP paste as a function of the initial cross-sectional area of the tracks. These samples were produced by increasing the print gap between the substrate and the nozzle from $100 \mu \mathrm{m}$ (for 1 layer) to $150 \mu \mathrm{m}$ (for 2 layers) and $200 \mu \mathrm{m}$ (for 3 layers). The samples were thermally cured after each layer. Nine tracks were prepared for each case and ultrasonically treated at medium level. The initial height of the tracks was approx. 14, 78 and $170 \mu \mathrm{m}$ for 1, 2 and 3 layers respectively, their width varied between 1.42 and $1.50 \mathrm{~mm}$ and their cross-sectional area between 0.02 and $0.26 \mathrm{~mm}^{2}$. Note that the cured surface of the tracks provided a better print surface, hence the second and third layers were thicker than the first. After the UAM treatment, the resistivity of the triple layer tracks increased up to 7 times more than the resistivity of the single layer tracks. With a cross-sectional area of $0.26 \mathrm{~mm}^{2}$, the triple layer Ag-TP tracks were thicker than any other of the printed tracks using C-TS, Cu-TS, Ag-TS2 or Ag-TS1 but the relative resistivity change was much lower than that of $\mathrm{Cu}-\mathrm{TS}$ or Ag-TS2. This indicates that the track thickness alone does not explain the very high resistivity change for $\mathrm{Cu}$-TS and Ag-TS2, however, taking the track thickness into account, the resistivity change after UAM 

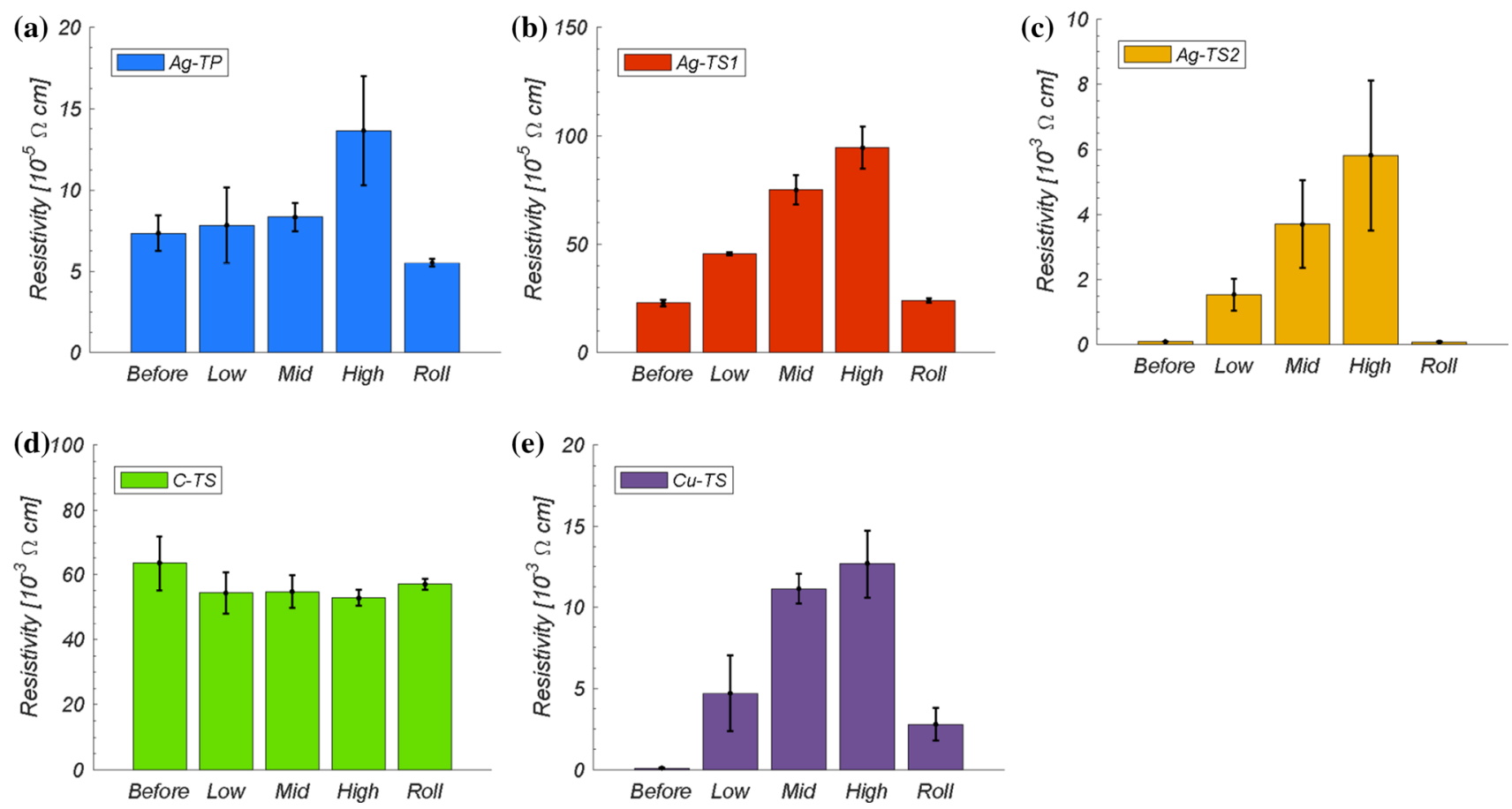

Fig. 5 The resistivity measured "before" treatment, after "low", "medium" and "high" energy UAM processing and after sonotrode "roll" without ultrasound for a Ag-TP, b Ag-TS1, c Ag-TS2, d C-TS, e Cu-TS

Fig. 6 The resistivity change versus UAM energy relative to the pre-treatment resistivity for a Ag-TP, Ag-TS1 and C-TS and b Ag-TS2 and $\mathrm{Cu}-\mathrm{TS}$
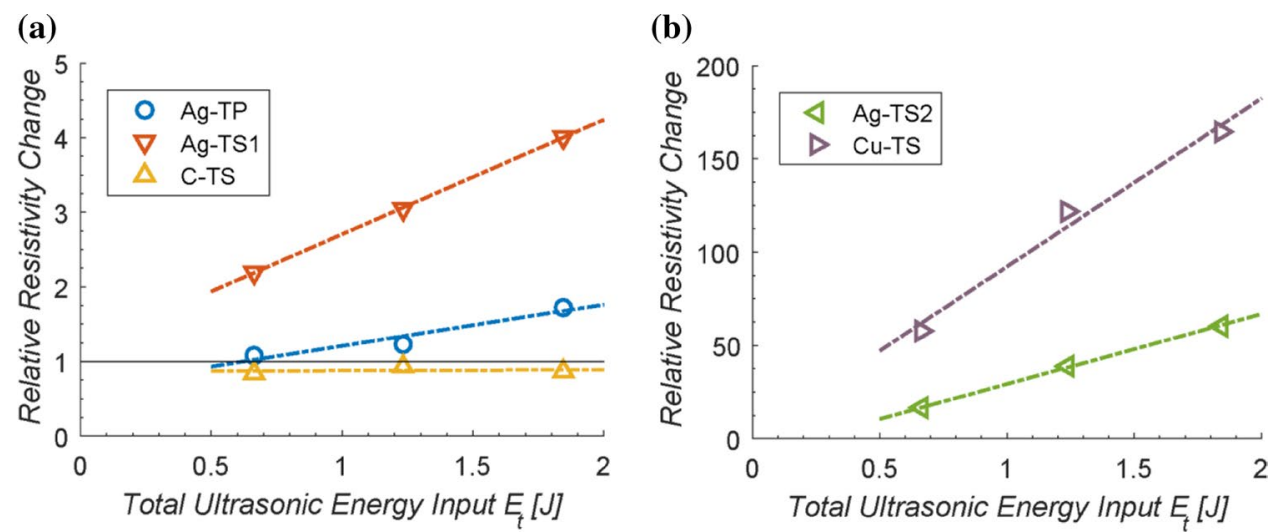

processing for the Ag-TP and Ag-TS1 pastes can be considered very similar. Notably, the initial resistivity of Ag-TP is significantly lower than that of Ag-TS1, so Ag-TP is still a more suitable material for UAM processing applications.

\subsection{Microstructure Analysis}

The microstructure of the printed tracks was examined using FIB-SEM imaging. The images in Figs. 8 and 9 show a cross-section of the printed tracks before and after UAM processing for each of the five pastes. Figure 8 shows the silver particles (light grey) embedded in the polymer matrix (dark grey). The carbon particles in Fig. 9a, b are visible as darker objects against a slightly brighter background, whereas the copper particles are clearly visible in Fig. 9c, d as semi-porous particles in the polymer binder.

A number of voids were observed in the cross-section of the Ag-TP sample (see Fig. 8a) before the ultrasonic treatment and to a lesser extent in the C-TS control sample (see Fig. 9a) and are highlighted as "dispensing defects" in the figures. A probable cause of these defects is the entrapment of air bubbles in the paste during loading of the syringe before dispensing. These defects are not uncommon during syringe dispensing [32]. The voids increase the apparent cross-sectional area measured through surface scanning, resulting in a higher bulk resistivity. The forces introduced during the rolling motion of the sonotrode can cause these voids to collapse though, compressing the samples, resulting 


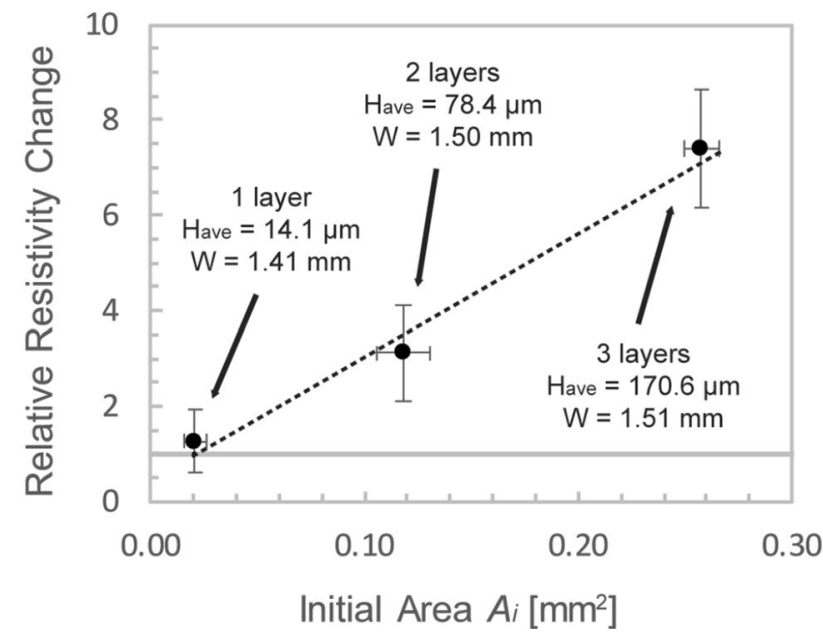

Fig. 7 Relative resistivity change for increasing cross-sectional area after UAM processing for printed Ag-TP tracks with 1, 2 and 3 layers of conductive paste

in lower cross-sectional area of the tracks. This can explain the measured decrease in resistivity and dimensions of the samples that were subjected to roll only.

In the ultrasonically treated Ag-TS2 sample (see Fig. 8f), a significant number of voids were observed between the silver particles and the polymer binder, where the particles have dislodged from the binder. Similar voids were not present in the control sample and it can be concluded that the ultrasonic treatment was the cause of these defects. The same material was used in the study of $\mathrm{Li}$ et al. [16], where dislodging of the filler particles from the surrounding resin was also reported. This suggests that the phenomenon might be material dependent and related to the material of the polymer binder. The creation of voids in Ag-TS2 during UAM processing also explains the difference in the cross-sectional area of these materials between the samples that were subjected to roll only and the UAM treated tracks. This indicates that the combination of ultrasonic oscillations and pressure, and not the pressure alone, is causing the void formation.

An interesting effect was observed in the case of Ag-TS1. In the control sample (Fig. 8c) the silver filler particles and flakes appeared to be randomly oriented, while in the ultrasonically treated sample (Fig. 8d) the particles had an almost homogeneous orientation. A possible cause of this change is the large degree of material flow induced during the ultrasonic treatment that was described previously. The Ag-TS1 tracks were the thickest and underwent the largest deformation leading to an increased probability of aligning the flakes.

A general observation is that the thermoset pastes with $\mathrm{Ag}$ and $\mathrm{Cu}$ particles are most affected by the ultrasound treatment (i.e. the $\mathrm{Cu}-\mathrm{TS}, \mathrm{Ag}-\mathrm{TS} 2$ and Ag-TS1 pastes), whereas the thermoplastic paste with $\mathrm{Ag}$ particles and the carbon based thermoset paste perform much better (i.e. the Ag-TP and C-TS pastes). This difference could be explained using percolation theory and taking into consideration the material changes taking place during UAM processing. The pastes used in this study are isotropic conductive adhesives (ICAs). During curing and subsequent cooling the polymer binder shrinks and pressure is applied between the filler particles. In ICAs there are three main contributors to electrical resistance [19]: the resistance of the bulk particle material $\left(\mathrm{R}_{\mathrm{p}}\right)$, the resistance due to contact $\left(\mathrm{R}_{\mathrm{cr}}\right)$ and tunnelling resistance $\left(\mathrm{R}_{\mathrm{t}}\right)$ when current propagates through the insulating binder, from one particle to the next, when they are in close proximity $(<10 \mathrm{~nm})$ but not in contact. During normal conditions, the main contributor to the resistance is the contact resistance, $R_{c r}$ and to a lesser degree, $R_{p}$. The UAM processing deforms the binder material, due to the generated heat and pressure. During this the thermoset binder undergoes a plastic deformation, breaking the cross-linked bonds between the polymer chains, which releases the internal pressure between the filler particles built up in the curing process and increases the contact resistance, $R_{\text {cr. }}$ In contrast, the thermoplastic binder can withstand the mechanical and thermal loads without significant degradation of its mechanical properties, as the energy and generated heat of the UAM process softens the binder. Thereby it can contract and deform, reapplying most of the inter-particle pressure, leading to a limited increase in contact resistance. From simulations [18], there is a linear correlation in the logarithmic scale between the inter-particle contact resistance and the resistivity of an ICA (for particles with aspect ratio of 2:1). Doubling the contact resistance increases the resistivity by approximately an order of magnitude. Also, the effect is larger in particles with a dimensional aspect ratio close to 1 . This may explain the large observed resistivity increase in Ag-TS1 and Cu-TS, which contain particles rather than flakes.

The manufacturers of the ICAs tested here do not give a particle size distribution or the dimensional aspect ratio of the conductive particles, but the FIB-SEM images in Figs. 8 and 9 provide qualitative information of the particles' shape and size, although not for C-TS. (Standard SEM images of the cured ICA surfaces were not able to distinguish individual particles as the particles form a continuous film along the surface). The particles in Ag-TP and Ag-TS2 are clearly flake-like with the shortest dimension in the region of $0.5-1 \mu \mathrm{m}$. Ag-TS1 contains similar flakes, but also a large quantity of elongated particles with the smallest dimension in the region of $2-3 \mu \mathrm{m}$. The particle distribution of $\mathrm{Cu}-\mathrm{TS}$ is significantly different as it only contains irregular shaped particles with an average diameter in the range of 14-25 $\mu \mathrm{m}$ and an aspect ratio close to 1 . These large rigid particles in $\mathrm{Cu}$-TS have very few contact points to create a conductive path through the paste and the contact points are likely to be 
Fig. 8 FIB-SEM images of a cross-section of Ag-TP, Ag-TS1 and Ag-TS2 conductive tracks before and after UAM processing
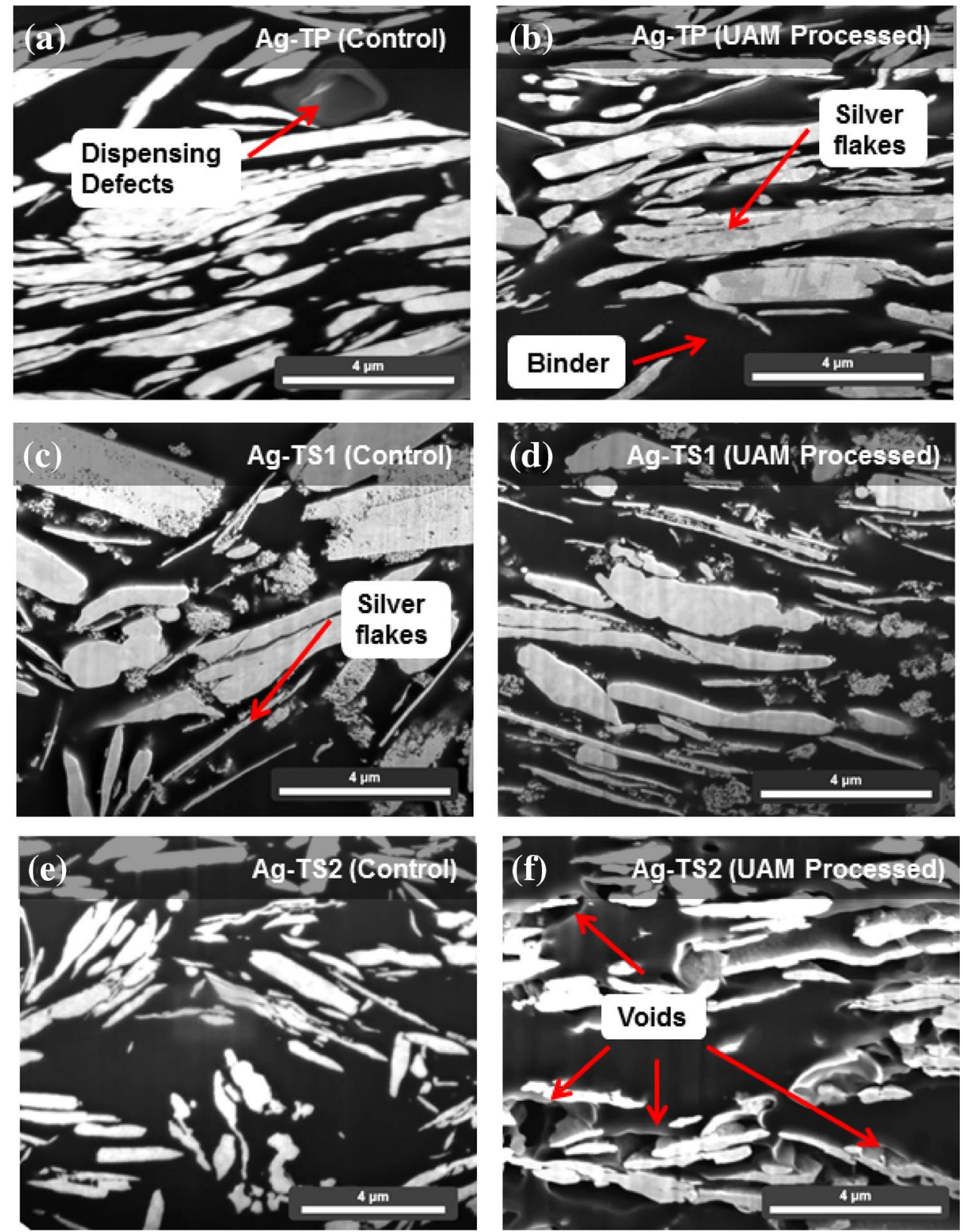

broken by the mechanical strain caused by the force applied by the sonotrode. The thermoset polymer matrix in $\mathrm{Cu}$-TS is not able to reflow around the particles and the broken contact points are less likely to get reconnected. The difference in particle shape and size is therefore likely the reason for the significant increase in the resistivity of $\mathrm{Cu}$-TS during "roll only" and generally being poorly compatible with UAM in this study.
Resistivity change can also originate from particle bonding. It was expected that ultrasonic welding of the metal particles would occur and perhaps increase the conductivity. Image analysis showed some evidence of particle bonding between the smaller particles for Ag-TP and Ag-TS2: the average particle size increased while the total particle count decreased, suggesting that the smaller particles created larger particle agglomerates. Nevertheless, 
Fig. 9 FIB-SEM images of a cross-section of C-TS and $\mathrm{Cu}$-TS conductive tracks before and after UAM processing
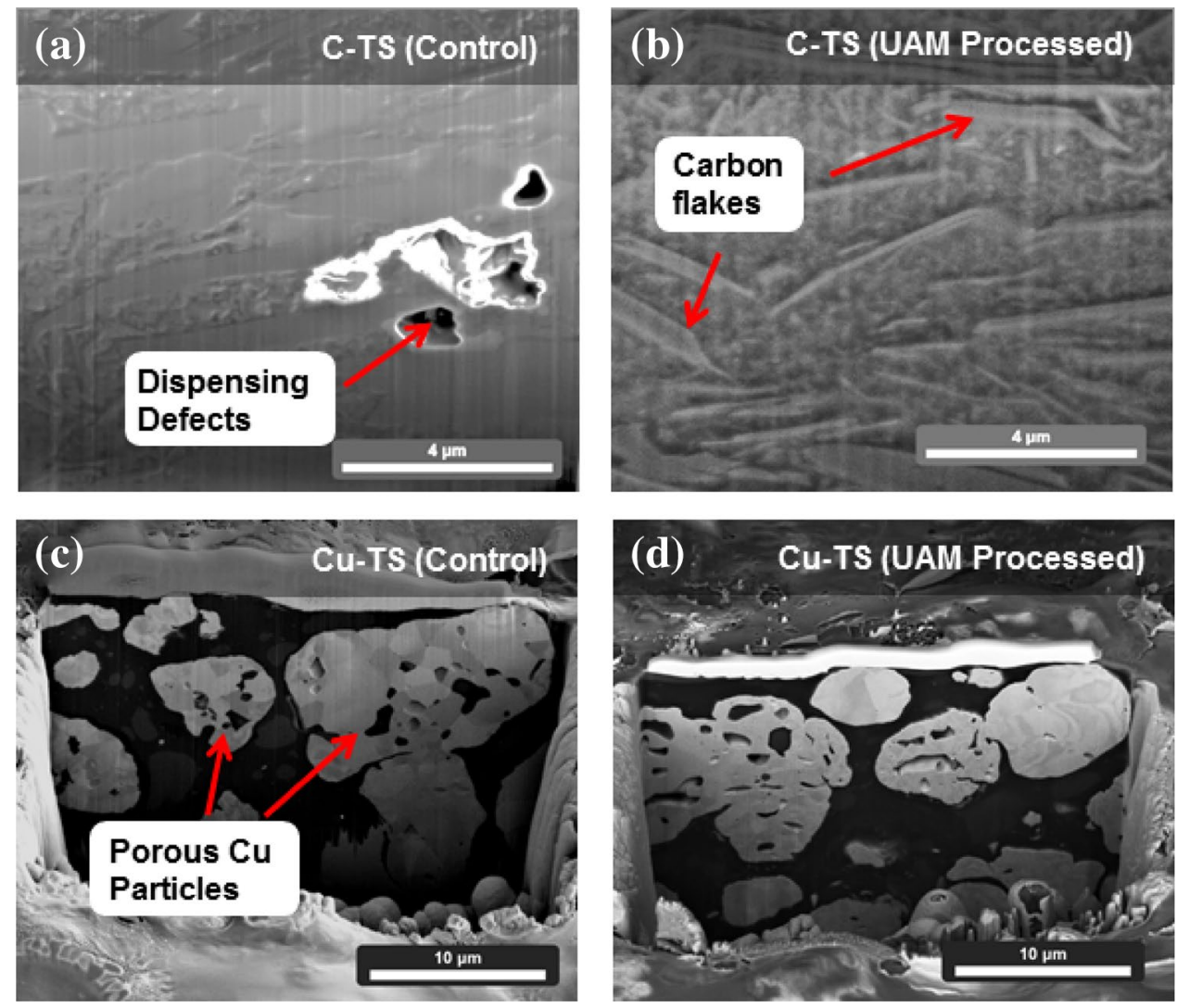

the small number of experimental data points (only one FIB-SEM cross-section for each material) did not allow for statistically significant conclusions to be made.

Unlike all metal-filled conductive materials, the microstructure of the C-TS sample remained unaltered after the ultrasonic treatment. As expected, there was no bonding between the carbon flakes, since carbon is not compatible with ultrasonic welding. In fact, very little evidence of any change in the microstructure of the examined sample could be identified (see Fig. 9b). No evidence of defects or cracks of the carbon flakes after the ultrasonic treatment were observed in the cross-section, while only minor micro-sized cracks were encountered on the surface of the treated sample. The excellent response of the carbon/graphite samples to the ultrasonic treatment make this material a very good candidate for fabricating printed electronic structures embedded in metal via UAM. However, the conductivity of the currently available carbon/ graphite pastes is two orders of magnitude lower than that of their silver-filled counterparts, deeming them unsuitable for the creation of electronic interconnects and more suitable for creating resistors. Nevertheless, future materials with low resistivity that use carbon nanotubes or graphene as the conductive filler would make excellent candidates for this application.

\section{Conclusion}

The effect of ultrasonic additive manufacturing on printed electronic tracks was investigated for five different conductive pastes. It was found that the type of polymer binder in the paste greatly affects the resistivity increase after ultrasound treatment and that the resistivity increase is proportional to the ultrasonic energy. The resistivity for a thermoset polymer binder with silver or copper particles/flakes (i.e. the Ag-TS1, Ag-TS2 and Cu-TS materials) could increase more than 150 times due to the conductive particles dislodging from the binder or the particle's contact points were broken thereby breaking the conductive path through the paste. The thermoplastic paste (Ag-TP) showed only a moderate resistivity change (up to $50 \%$ ), possibly due to particle agglomeration of the smaller filler particles. However, particle dislodging and void formation was avoided due to the flow of the thermoplastic binder during the ultrasonic treatment allowing the conductive path to be recreated. The thickness of the printed tracks affected the resistivity adversely, due to an increased deformation during UAM processing. The conductive tracks printed with the carbon flake/particle based thermoset paste (C-TS) were unaffected by the ultrasonic treatment. Although the carbon-based paste is the most 
stable under UAM conditions, its initial resistivity before treatment is 2-3 orders of magnitude higher than the $\mathrm{Ag}$ and $\mathrm{Cu}$ based pastes, making an $\mathrm{Ag}$ particle/flake based thermoplastic paste, such as the Ag-TP material, the most suitable paste for conductive tracks embedded using UAM. Alternative methods to limit the damage to the conductive tracks would be to reduce the ultrasonic energy or printing the conductive tracks in grooves, but as both of these approaches limit the quality of the ultrasonic welding [15], choosing an appropriate paste for the conductive tracks is essential.

Acknowledgements The authors acknowledge use of facilities within the Loughborough Materials Characterisation Centre.

Open Access This article is distributed under the terms of the Creative Commons Attribution 4.0 International License (http://creativeco mmons.org/licenses/by/4.0/), which permits unrestricted use, distribution, and reproduction in any medium, provided you give appropriate credit to the original author(s) and the source, provide a link to the Creative Commons license, and indicate if changes were made.

\section{References}

1. Leigh, S.J., Bradley, R.J., Purssell, C.P., Billson, D.R., Hutchins, D.A.: A simple, low-cost conductive composite material for 3D printing of electronic sensors. PLoS ONE 7, e49365 (2012)

2. Friel, R.J.: Power ultrasonics for additive manufacturing and consolidating of materials. In: Gallego-Juárez, J.A., Graff, K.F. (eds.) Power Ultrasonics - Applications of High-Intensity Ultrasound, pp. 313-335. Woodhead Publishing, Cambridge (2015)

3. Kong, C.Y., Soar, R.C., Dickens, P.M.: Optimum process parameters for ultrasonic consolidation of 3003 aluminium. J. Mater. Process. Technol. 146, 181 (2004)

4. Kong, C.Y., Soar, R.C., Dickens, P.M.: A model for weld strength in ultrasonically consolidated components. Proc. Inst. Mech. Eng. Part C J. Mech. Eng. Sci. 219, 83 (2005)

5. Kong, C.Y., Soar, R.: Method for embedding optical fibers in an aluminum matrix by ultrasonic consolidation. Appl. Opt. 44, 6325 (2005)

6. Monaghan, T., Capel, A.J., Christie, S.D., Harris, R.A., Friel, R.J.: Solid-state additive manufacturing for metallized optical fiber integration. Compos. Part A Appl. Sci. Manuf. 76, 181 (2015)

7. Friel, R.J., Harris, R.A.: A nanometre-scale fibre-to-matrix interface characterization of an ultrasonically consolidated metal matrix composite. Proc. Inst. Mech. Eng. Part L J. Mater. Des. Appl. 224, 31 (2010)

8. Masurtschak, S., Friel, R.J., Harris, R.A.: New concept to aid efficient fibre integration into metal matrices during ultrasonic consolidation. Proc. Inst. Mech. Eng. Part B J. Eng. Manuf. 231, 954405415592120 (2015)

9. Hahnlen, R., Dapino, M.J.: Active metal-matrix composites with embedded smart materials by ultrasonic additive manufacturing. In: McMickell, M.B., Farinholt, K.M. (eds.) Proceedings of SPIE-International Society for Optics Engineering, p. 764500. International Society for Optics and Photonics (2010)

10. Wolcott, P.J., Hopkins, C.D., Zhang, L., Dapino, M.J.: Smart switch metamaterials for multiband radio frequency antennas. J. Intell. Mater. Syst. Struct. 22, 1469 (2011)
11. Sriraman, M.R., Gonser, M., Fujii, H.T., Babu, S.S., Bloss, M.: Thermal transients during processing of materials by very high power ultrasonic additive manufacturing. J. Mater. Process. Technol. 211, 1650 (2011)

12. Schick, D., Suresh Babu, S., Foster, D.R., Dapino, M., Short, M., Lippold, J.C.: Transient thermal response in ultrasonic additive manufacturing of aluminum 3003. Rapid Prototyp. J. 17, 369 (2011)

13. Robinson, C.J., Stucker, B., Lopes, A.J., Wicker, R.: Integration of Direct Write and Ultrasonic Consolidation Technologies to Create Advanced Structures with Embedded Electrical Circuitry. 17th Solid Freeform Fabrication Symposium, pp. 60-69. Austin, TX (2006)

14. Siggard, E.J., Madhusoodananb, A.S., Stucker, B., Eames, B.: Structurally embedded electrical systems using ultrasonic consolidation (UC) 70. In: Proceedings of the 17th Annual Solid Freeform Fabrication Symposium, pp. 70-83. Austin (2006)

15. Li, J., Monaghan, T., Masurtschak, S., Bournias-Varotsis, A., Friel, R.J., Harris, R.A.: Exploring the mechanical strength of additively manufactured metal structures with embedded electrical materials. Mater. Sci. Eng. A 639, 474 (2015)

16. Li, J., Monaghan, T., Nguyen, T.T., Kay, R.W., Friel, R.J., Harris, R.A.: Multifunctional metal matrix composites with embedded printed electrical materials fabricated by ultrasonic additive manufacturing. Compos. Part B Eng. 113, 342 (2017)

17. Bournias-Varotsis, A., Harris, R.A., Friel, R.J.: The effect of ultrasonic excitation on the electrical properties and microstructure of printed electronic conductive inks. In: 2015 38th International Spring Seminar on Electronics Technology, pp. 140-145. IEEE (2015)

18. Li, L., Morris, J.E.: Electrical conduction models for isotropically conductive adhesive joints. IEEE Trans. Compon. Packag. Manuf. Technol. Part A 20, 3 (1997)

19. Lu, D., Tong, Q.K., Wong, C.P.: Conductivity mechanisms of isotropic conductive adhesives (ICAs). IEEE Trans. Electron. Packag. Manuf. 22, 223 (1999)

20. Ruschau, G.R., Yoshikawa, S., Newnham, R.E.: Percolation constraints in the use of conductor-filled polymers for interconnects. In: Proceedings of the 42nd Electronic Components and Technology Conference, pp. 481-486. IEEE (1992)

21. Qi, S., Vaidhyanathan, B., Hutt, D.: Conventional and microwaveassisted processing of $\mathrm{Cu}$-loaded ICAs for electronic interconnect applications. J. Mater. Sci. 48(20), 7204-7214 (2013)

22. Gwent Group: Conductive Silver Polymer Paste C2110817D5 (2017)

23. EPO-TEK: Conductive Epoxy E4110-PFC (2017)

24. Gwent Group, Flexible Silver Ink C2131014D3 (2017)

25. Gwent Group: Heat Curable Carbon Ink C2050503P1 (2017)

26. Bournias-Varotsis, A., Friel, R.J., Harris, R.A., Engstrom, D.: Selectively anodised aluminium foils as an insulating layer for embedding electronic circuitry in a metal matrix via ultrasonic additive manufacturing. In: Proceedings of the 26th Annual International SFF Symposium, pp. 2260-2270. Austin, TX (2016)

27. Kong, C.Y., Soar, R.C., Dickens, P.M.: Ultrasonic consolidation for embedding SMA fibres within aluminium matrices. Compos. Struct. 66, 421 (2004)

28. Yang, Y., Ram, G.D.J., Stucker, B.E.: An analytical energy model for metal foil deposition in ultrasonic consolidation. Rapid Prototyp. J. 16, 20 (2010)

29. Happonen, T., Voutilainen, J.-V., Fabritius, T.: Reliability study on adhesive interconnections in flex-to-flex printed electronics applications under environmental stresses. IEEE Trans. Device Mater. Reliab. 14, 1005 (2014)

30. Paul, G., Torah, R., Yang, K., Beeby, S., Tudor, J.: An investigation into the durability of screen-printed conductive tracks on textiles. Meas. Sci. Technol. 25, 25006 (2014) 
31. Ahn, B.Y., Duoss, E.B., Motala, M.J., Guo, X., Park, S.-I., Xiong, Y., Yoon, J., Nuzzo, R.G., Rogers, J.A., Lewis, J.A.: Omnidirectional printing of flexible, stretchable, and spanning silver microelectrodes. Science 80, 323 (2009)
32. Li, J., Wasley, T., Nguyen, T.T., Ta, V.D., Shephard, J.D., Stringer, J., Smith, P., Esenturk, E., Connaughton, C., Kay, R.: Hybrid additive manufacturing of 3D electronic systems. J. Micromech. Microeng. 26, 105005 (2016) 